\title{
Moxetumomab pasudotox-tdfk for relapsed/refractory hairy cell leukemia: a review of clinical considerations
}

\author{
Carmen F. Nobre ${ }^{1}\left[\right.$ D $\cdot$ Matthew J. Newman ${ }^{1}\left[\right.$ [ $\cdot$ Anne DeLisa ${ }^{1} \cdot$ Pauline Newman $^{1}$
}

Received: 10 April 2019 / Accepted: 14 May 2019 / Published online: 27 May 2019

(c) The Author(s) 2019

\begin{abstract}
Purpose Hairy cell leukemia (HCL) is a rare mature B cell leukemia. Purine analogs are the mainstay of treatment of HCL, but relapse after purine analog therapy is common. Outcomes of treatment of relapsed/refractory HCL typically diminish with each successive line of therapy. Moxetumomab pasudotox-tdfk is a novel recombinant immunotoxin approved for the treatment of patients with relapsed/refractory HCL who have received at least two prior therapies, including a purine analog. This article reviews HCL treatment, focusing on moxetumomab pasudotox-tdfk, its place in therapy, considerations for preparation and administration, and strategies for prevention and management of toxicities.

Methods A literature search was conducted in the PubMed database from inception to January 2019, using the following terms: moxetumomab, hairy cell leukemia, relapsed/refractory hairy cell leukemia, immunotoxin, and CD22. The package insert and available posters and abstracts were also reviewed.

Results FDA approval of moxetumomab pasudotox-tdfk was based on a phase III single-arm, open-label trial in 80 patients. Treatment with moxetumomab pasudotox-tdfk yielded a durable complete response rate of $30 \%$ with a median duration of response that had not yet been reached at a median follow-up of 16.7 months. The objective response rate was $75 \%$ based on blinded independent central review. The most common adverse reactions were infusion-related reactions, edema, nausea, fatigue, headache, pyrexia and anemia. Serious adverse events include capillary leak syndrome and hemolytic uremic syndrome.

Conclusions Clinicians providing care for patients receiving moxetumomab pasudotox-tdfk should be aware of the strategies required for safe administration, including the management of serious adverse events.
\end{abstract}

Keywords Hairy cell leukemia $\cdot$ CD22 Immunotoxin $\cdot$ Moxetumomab pasudotox-tdfk

$\begin{array}{ll}\text { Abbreviations } \\ \text { BCR } & \text { B-cell receptor } \\ \text { CLS } & \text { Capillary leak syndrome } \\ \text { CR } & \text { Complete response } \\ \text { FDA } & \text { Food and drug administration } \\ \text { HCL } & \text { Hairy cell leukemia } \\ \text { HUS } & \text { Hemolytic uremic syndrome } \\ \text { IRR } & \text { Infusion-related reactions } \\ \text { MRD } & \text { Minimal residual disease }\end{array}$

Carmen F. Nobre

cnobre1@jhmi.edu

1 Department of Pharmacy, The Johns Hopkins Hospital, Baltimore, MD 21287, USA

\section{Description and epidemiology}

Hairy cell leukemia (HCL) is a rare, chronic, mature B-cell malignancy occurring in an estimated 1100 patients annually in the United States [1]. The disease is characterized by the presence of lymphocytes with cytoplasmic projections appearing hair-like by microscopy, which infiltrate the bone marrow and spleen resulting in cytopenias, bone marrow fibrosis, and splenomegaly [2]. HCL comprises only $2 \%$ of all leukemias, the median age at diagnosis of $\mathrm{HCL}$ is 50 years, and the disease has a $4: 1$ male predominance [3]. Environmental exposures associated with farming, pesticides, and ionizing radiation may be risk factors [4-6]. Outcomes are typically positive, with $78 \%$ of patients alive at 15 years; overall survival is $96 \%$ when censoring for deaths unrelated to HCL [7]. However, relapses occur in about half of patients initially treated with a purine analog after 16 years of follow-up, and outcomes including rate 
of complete response and duration of relapse-free survival are diminished with each successive line of treatment [7]. The 2008 World Health Organization classification recognized the classic form of HCL as distinct from the rarer variant form, which accounts for $10-20 \%$ of all HCL cases [8]. HCL variant is characterized by a poorer response to purine analog monotherapy, increased lymphocytosis, and cytopenias without monocytopenia, as compared with the classic form [9].

\section{Pathophysiology}

Malignant cells infiltrate the bone marrow in nearly all patients with HCL [10]. Other affected organs include the spleen, liver, and lymph nodes [11]. Flow cytometry on peripheral blood or bone marrow aspirate in HCL will be positive for CD11c, CD19, CD20, CD22, CD25, CD103, CD123, cyclin D1, annexin A1, and CD200, and negative for CD5 and CD10 [12]. HCL variant is negative for CD25, $\mathrm{CD} 123$, and annexin A1 [12]. The BRAF V600E mutation is present in most cases of the classic form of HCL but absent in the variant [13]. Molecular analysis for the IGHV4-34 rearrangement may be employed. HCL expressing the IGHV4-34 rearrangement shares clinical features of the variant (including poorer response to purine analogs and poorer prognosis) and lacks BRAF mutation, but may be classified as classic HCL by immunophenotype $[8,11]$.

\section{Clinical characteristics}

Patients with HCL present most commonly with fatigue, recurrent infections, and symptomatic or asymptomatic splenomegaly, with other symptoms including weakness, fevers, night sweats, weight loss, and bruising [8, 11]. Initial treatment of HCL is indicated in the presence of symptomatic disease, or cytopenias (hemoglobin $<11 \mathrm{~g} /$ $\mathrm{dL}$, platelets $<100,000 / \mu \mathrm{L}$ and/or absolute neutrophil count $[\mathrm{ANC}]<1000 / \mu \mathrm{L}$ ), progressive lymphocytosis, or lymphadenopathy [14]. If the patient is asymptomatic, a reasonable approach is close observation with initial treatment upon development of symptoms [11].

\section{Review of treatment}

Historically, HCL was managed with interferon and splenectomy [2]. These treatment approaches have been largely replaced by the purine analogs, pentostatin or cladribine. An early Eastern Cooperative Oncology Group study of pentostatin in untreated and previously treated HCL (including splenectomy, chemotherapy, or both) demonstrated a response rate of $96 \%$ and a complete response (CR) rate of $59 \%$ [15]. A randomized trial of pentostatin versus interferon alfa-2a in newly diagnosed HCL resulted in improved CR rates with pentostatin ( $76 \%$ vs. $11 \%$, respectively), with improvements in response rate and relapse-free survival [16]. In a more recent long-term follow-up study of patients who received pentostatin, estimated survival rates were $90 \%$ at 5 years and $81 \%$ at 10 years, with two of 40 deaths attributed to HCL during the follow-up period [17].

Outcomes of treatment with cladribine in patients with HCL are described in Table 1. The majority of patients attain a CR, and, although overall survival is prolonged, a significant proportion will relapse and require additional therapy.

A retrospective review of 233 patients with HCL did not find any clinically relevant differences in outcomes between those treated with pentostatin and those treated with cladribine, including CR rate ( $82 \%$ vs. $76 \%$, not significant $[\mathrm{NS}]$ ), and the two are considered interchangeable for first- and second-line therapy [7]. The purine analogs are associated with profound neutropenia, reduction in CD4 + T cells, and resultant opportunistic infections [14].

For patients achieving a CR (hemoglobin $>11 \mathrm{~g} / \mathrm{dL}$ without transfusion, platelets $>100,000 / \mu \mathrm{L}, \mathrm{ANC}>1500 / \mu \mathrm{L}$, regression of splenomegaly on examination, and absence of morphologic HCL on peripheral blood smear and in bone marrow), observation is employed to detect relapse. Treatment options for patients relapsing 2 years or more after achieving a CR include retreatment with the original agent or the alternative purine analog with or without the addition

Table 1 Outcomes after initial treatment with cladribine

\begin{tabular}{|c|c|c|c|c|c|c|}
\hline Study & Patients, $n$ & Dosing & $\mathrm{CR}, \%$ & $\mathrm{PR}, \%$ & Relapse & OS \\
\hline Estey et al. [53] & 46 & $4 \mathrm{mg} / \mathrm{m}^{2}$ daily CIV for 7 days & 78 & 11 & $\begin{array}{l}\text { One patient at } 71 \text { weeks (median } \\
\text { follow-up } 37 \text { weeks) }\end{array}$ & Not evaluated \\
\hline Saven et al. [54] & 349 & $0.1 \mathrm{mg} / \mathrm{kg}$ daily CIV for 7 days & 91 & 7 & $\begin{array}{r}26 \% \text { at median } 29 \text { months; } 62 \% \\
\text { achieved CR with retreatment }\end{array}$ & $96 \%$ at 48 months \\
\hline Goodman et al. [18] & 207 & $0.1 \mathrm{mg} / \mathrm{kg}$ daily CIV for 7 days & 95 & 5 & $37 \%$ at median 42 months & $97 \%$ at 108 months \\
\hline Chadha et al. [55] & 86 & $0.1 \mathrm{mg} / \mathrm{kg}$ daily CIV for 7 days & 79 & 21 & $\begin{array}{l}36 \% \text { at median } 9.7 \text { years; } 52 \% \text { achieved } \\
\text { CR with retreatment }\end{array}$ & $87 \%$ at 12 years \\
\hline
\end{tabular}

$C I V$ continuous intravenous infusion, $C R$ complete response, $O S$ overall survival, $P R$ partial response 
of rituximab. Of patients with relapsed disease who were retreated with cladribine, the rate of second CR was $75 \%$ with a median response of 35 months [18]. Single-agent rituximab may be considered for those unable to tolerate a purine analog. If relapse occurs within 2 years, or a suboptimal response (less than a CR) is achieved with initial therapy, recommendations include a clinical trial, the alternative purine analog with or without rituximab, interferon alfa, rituximab monotherapy, or vemurafenib. In the setting of progression after second-line treatment, options prior to the Food and Drug Administration (FDA) approval of moxetumomab pasudotox-tdfk included a clinical trial, vemurafenib with or without rituximab, or ibrutinib [11].

Two smaller studies established the combination of rituximab and a purine analog for relapsed HCL. In the first, all 14 patients achieved CR and were alive at 5 years' followup [19]. A second retrospective study resulted in a CR rate of $89 \%$, which was maintained at a median follow-up of 36 months [20]. Rituximab monotherapy is somewhat less active than the combination of rituximab and a purine analog, with CRs ranging from 10 to 53\% [21-24]. It is recommended mainly for patients unable to receive a purine analog [11].

Newer oral agents have been used effectively in relapsed/ refractory (R/R) HCL. Vemurafenib has resulted in an overall response rate of $100 \%$ and a CR in $42 \%$ of patients at 12 weeks [25]. At 1 year, progression-free survival was 73\%, and overall survival was $91 \%$. Additionally, median relapsefree survival was 9 months in responders and 19 months for those with a CR. Toxicities included rash, arthralgias or arthritis, pyrexia, and elevated bilirubin. The addition of rituximab to vemurafenib yielded an observed CR rate of $100 \%$ in 25 evaluable patients, with $70 \%$ of patients achieving CR after 4 weeks of treatment [26]. Ibrutinib has been evaluated mainly in patients with relapsed classical and variant HCL previously treated with a purine analog. The objective response rate was $46 \%$ and more common in patients with classical HCL [27]. Progression-free survival at 24 months was $79 \%$. Toxicities included grade 3 or higher lymphopenia (21\%), neutropenia (18\%), thrombocytopenia $(14 \%)$, and infections in $25 \%$ of patients.

The risk of relapse in HCL has been proposed using the following categories based on minimal residual disease (MRD): $<1 \%$ positive cells, low risk; $1-5 \%$ intermediate risk; $>5 \%$ higher risk [28]. In patients treated with cladribine followed by rituximab, MRD was undetectable in 92-94\% $[19,29]$. In patients treated with vemurafenib and rituximab, MRD was undetectable in $73 \%$ of evaluated patients [30].

Moxetumomab pasudotox-tdfk (LUMOXITI ${ }^{\circledR}$, AstraZeneca Pharmaceuticals LP) is a novel recombinant CD22directed cytotoxin targeting CD22, which has been newly approved for the treatment of patients with R/R HCL who have received at least two prior therapies, including at least one purine analog [31]. This is the first FDA-approved therapy for R/R HCL since cladribine in 1993. Clinicians should be aware of the preparation, administration, monitoring, and adverse-effect management strategies necessary to ensure safe and effective use of moxetumomab pasudotox-tdfk.

\section{Immunotoxins and CD22}

Immunotoxins are useful in delivering targeted anticancer therapy. They are proteins that consist of a targeting portion, such as an antibody or growth factor, linked to a toxin. Toxins used in immunotoxin products are procured from bacteria, fungi, and plants, and most function by inhibiting protein synthesis. Commonly used bacterial toxins include diphtheria toxin and Pseudomonas exotoxin (PE) [32]. Recombinant immunotoxins contain an $\mathrm{Fv}$ fragment fused to a truncated protein toxin [33]. Agents tested in HCL include LMB-2 targeting CD25 and BL22/CAT-3888 targeting CD22. BL22/ CAT-3888 consisted of disulfide-linked VH and VL chains of the murine anti-CD22 monoclonal antibody RFB4 fused to a truncated form of exotoxin A, PE38 [34, 35]. BL22/ CAT-3888 served as the precursor to CAT-8015/moxetumomab pasudotox-tdfk.

CD22 is a B-lymphocyte lineage-restricted transmembrane protein that first emerges on the face of pre-B-cells and is fully expressed by differentiated $\operatorname{IgM}^{+}, \operatorname{IgD}^{+} \mathrm{B}$-cells [36-39]. The cytoplasmic domain of CD22 has six tyrosines as potential targets for phosphorylation, with regions related to the tyrosine-based inhibition motif $[38,39]$. Tyrosinebased inhibition motifs are also substrates for Src family tyrosine kinases, and docking sites for $\mathrm{SH} 2$ domains, including SHP-1. Association with these inhibitory proteins results in negative regulation of B-cell receptor (BCR) signaling, thus leading to apoptosis of B-cells [40, 41]. CD22 is rapidly phosphorylated after BCR cross-linking. It has a basal halflife of about $8 \mathrm{~h}$ and is internalized in less than $1 \mathrm{~h}$ once it is bound by a ligand or cross-linked, and it does not recycle to the cell surface from the intracellular pool. Once endocytosed, CD22 is directed to the lysosome for degradation [39]. CD22 is an attractive target for immunotoxin therapy, as it is a B-cell antigen expressed particularly strongly in HCL [42].

Moxetumomab pasudotox-tdfk (CAT-8015) is a second-generation recombinant immunotoxin, with increased affinity compared with BL22 [43]. Affinity for CD22 was increased 14-fold by hot-spot mutagenesis [44]. Moxetumomab pasudotox-tdfk contains threonine-histidine-tryptophan instead of serine-serine-tyrosine in the antigen-binding site of the heavy chain [45]. It is formed of a murine immunoglobulin variable domain genetically fused to a truncated form of Pseudomonas exotoxin, PE38 [31, 46].

After binding to CD22, moxetumomab pasudotox-tdfk is internalized, and the Pseudomonas exotoxin catalyzes 
inhibition of protein synthesis by ADP-ribosylation of elongation factor 2, resulting in apoptotic cell death (Fig. 1) [31].

\section{Clinical results}

In an open-label phase 3 trial, 80 patients with R/R HCL with at least two prior therapies, including at least one purine analog, received moxetumomab pasudotox-tdfk $0.4 \mathrm{mg} / \mathrm{kg} /$ dose intravenously (IV) on days 1, 3, and 5 [47] of each 28 day cycle for up to six cycles or until disease progression or unacceptable toxicity [47]. At a median follow-up time of 16.7 months (range 2-49), durable CR, defined as the maintenance of hematologic remission $(\mathrm{Hgb} \geq 11 \mathrm{~g} / \mathrm{dL}$, neutrophils $\geq 1500 / \mathrm{mm}^{3}$, and platelets $\geq 100,000 / \mathrm{mm}^{3}$ without transfusions or growth factor for at least 4 weeks) more than 180 days after IRC-assessed CR, was achieved in 30\%, CR in $41 \%$, PR in $34 \%$, and objective response in $75 \%$, with $80 \%$ achieving hematologic remission. Adverse effects included peripheral edema (39\%), nausea (35\%), fatigue (34\%), and headache $(33 \%)$. Serious adverse effects included hemolytic uremic syndrome (HUS) (7.5\%) and capillary leak syndrome (CLS) (5\%). Neutropenia and decreased neutrophil count occurred infrequently $(5 \%$ and $7.5 \%$ of patients, respectively).

Results of 33 patients from the long-term follow-up dose escalation phase 1 study of patients with R/R HCL treated with moxetumomab pasudotox-tdfk $0.05 \mathrm{mg} / \mathrm{kg} / \mathrm{dose}$ on days 1,3 , and 5 every 28 days were CR in $64 \%$ (median CR duration: 42.4 months) and overall response in $88 \%$ of patients [48]. Flow cytometric analysis of bone marrow aspirate was performed for MRD assessment. The median CR duration was longer in patients with MRD-negative status $(n=11)$ than in those with MRD-positive status $(n=9)$ (42.1 months vs. 13.5 months; $P<0.001$ ). Most patients achieving MRD negativity remained in CR (10 patients), of whom 9 patients were without MRD at the time of study conclusion. Although the significance of MRD in HCL is not yet clearly defined, outcomes appear to be improved in patients achieving MRD negativity [49-51].

\section{Dosing and pharmacokinetics}

The labeled dose and schedule of moxetumomab pasudotoxtdfk are $0.04 \mathrm{mg} / \mathrm{kg} /$ dose, using actual body weight, on days 1,3 , and 5 of a 28 -day cycle. A change in dose should not be made during a cycle. Dose changes should only be made between cycles when a change in weight of greater than $10 \%$ is observed from the weight used to calculate the first dose of the first treatment cycle. Treatment should be continued for up to six cycles unless there is disease progression or severe toxicity including CLS or HUS. Moxetumomab pasudotoxtdfk was not studied in patients with moderate to severe hepatic impairment or severe renal impairment and should
Fig. 1 Moxetumomab pasudotox-tdfk mechanism of action. Reproduced from: https ://www.cancer.gov/news-event s/cancer-currents-blog/2018/ moxetumomab-fda-hairy-cellleukemia

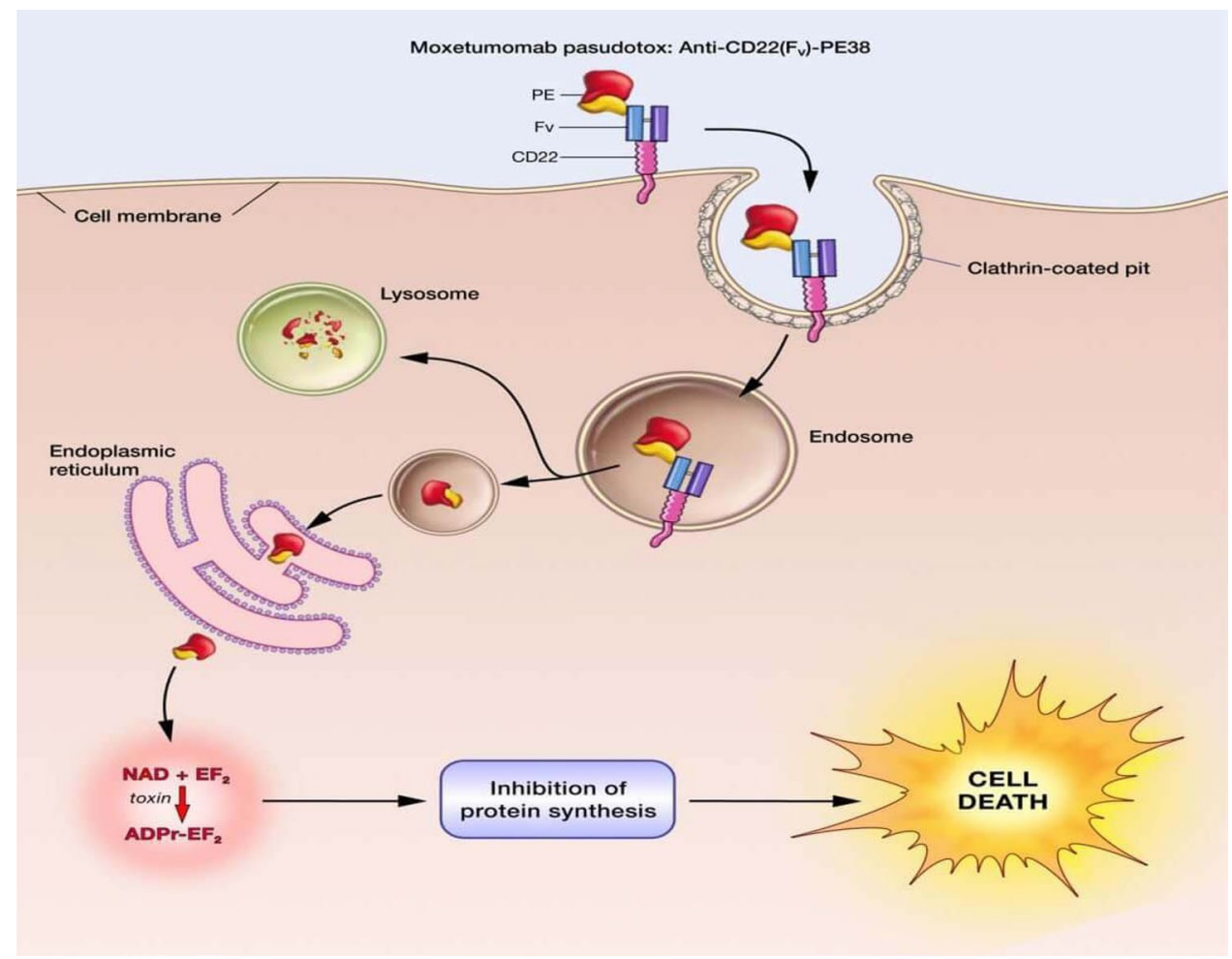


not be used in patients with creatinine clearance $\leq 29 \mathrm{~mL} /$ $\min [31]$.

Moxetumomab pasudotox-tdfk may interfere with detection of cellular CD22; consequently, total peripheral blood B-cell counts were measured using a surrogate of CD19+ B-cell assay. Circulating CD19+B-cells were reduced by $89 \%$ from baseline following the first three infusions of moxetumomab pasudotox-tdfk, and this was maintained for a minimum of 1 month post treatment. The B-cell count recovered approximately 6 months after the end of therapy. Additionally, a low baseline CD19+B-cell count was found to be significantly associated with a high pharmacokinetic exposure $(P<0.001)$.

Overall, the concentration of moxetumomab pasudotoxtdfk was found to increase in proportion to the dose. The half-life of moxetumomab pasudotox-tdfk is $1.4 \mathrm{~h}$ with a mean estimated systemic clearance of $25 \mathrm{~L} / \mathrm{h}$ after the first dose of the first cycle and decreasing to $4 \mathrm{~L} / \mathrm{h}$ after subsequent doses. No systemic accumulation of moxetumomab pasudotox-tdfk was observed. Moxetumomab pasudotoxtdfk is believed to undergo proteolytic degradation into small peptides and amino acids via catabolic pathways during metabolism, but the exact mechanism is unknown [31].

\section{Dose preparation}

It is important to understand the proper storage and preparation of moxetumomab pasudotox-tdfk. It is available as a single-dose vial containing $1 \mathrm{mg}$ of preservative-free lyophilized drug powder. The IV solution stabilizer is a singledose vial containing $1 \mathrm{~mL}$ of sterile, preservative-free solution, which is in a separate package from the moxetumomab pasudotox-tdfk lyophilized powder. Both the moxetumomab pasudotox-tdfk vials and the IV solution stabilizer should be stored in the refrigerator within their original cartons, protected from light, and should not be frozen. Moxetumomab pasudotox-tdfk is manufactured through a fermentation process in a nutrient medium composed of kanamycin, an aminoglycoside antibiotic. However, it should be noted that kanamycin is cleared from the manufacturing process and that it is not detectable in the final product [31].

To prepare, calculate the dose of moxetumomab pasudotox-tdfk using the patient's actual body weight and collect the number of vials needed for the dose preparation from the refrigerator. Each vial delivers $1 \mathrm{mg}$ of moxetumomab pasudotox-tdfk and is reconstituted with $1.1 \mathrm{~mL}$ of sterile water for injection, resulting in a concentration of $1 \mathrm{mg} / \mathrm{mL}$. Use partial vials when necessary to avoid rounding doses down. Gently rotate or swirl for $60 \mathrm{~s}$ or until the powder is dissolved. Do not shake the solution [31].
The IV infusion is prepared by first adding $1 \mathrm{~mL}$ of the IV solution stabilizer to a $50 \mathrm{~mL}$ infusion bag of $0.9 \%$ sodium chloride. It is important to note that only $1 \mathrm{~mL}$ of IV solution stabilizer is needed to prepare a single dose of moxetumomab pasudotox-tdfk, regardless of the dose or number of vials of drug used. The infusion bag should be inverted several times to ensure that the IV solution stabilizer is adequately mixed with the sodium chloride solution. The calculated dose volume of moxetumomab pasudotox-tdfk is withdrawn from the moxetumomab pasudotox-tdfk vial(s) and is added to the sodium chloride bag containing the IV solution stabilizer. The prepared infusion bag should be gently inverted several times, but not shaken, to mix the solution. The prepared infusion bag is stable for $4 \mathrm{~h}$ at room temperature, or $24 \mathrm{~h}$ if refrigerated at $2-8{ }^{\circ} \mathrm{C}\left(36-46^{\circ} \mathrm{F}\right)$, and should be protected from light [31]. If refrigerated, the diluted solution may be allowed to equilibrate at room temperature for a maximum of $4 \mathrm{~h}$ prior to administration. Reconstituted solution should not be stored and must be used immediately for dose preparation [31]. Institutional guidelines for safe storage and handling of hazardous substances should be followed.

\section{Administration and supportive measures}

Moxetumomab pasudotox-tdfk solution is to be infused over $30 \mathrm{~min}$. Prior to each infusion, patients over $50 \mathrm{~kg}$ should receive $1 \mathrm{~L}$ of hydration with an isotonic IV solution (e.g. $5 \%$ dextrose injection, $0.45 \%$ or $0.9 \%$ sodium chloride injection) over $2-4 \mathrm{~h}$. For patients under $50 \mathrm{~kg}$, administer $0.5 \mathrm{~L}$ of hydration.

Premedications are to be administered 30-90 min prior to the infusion with an antihistamine, acetaminophen, and a histamine-2 receptor antagonist.

The antihistamine and antipyretics may be continued for up to $24 \mathrm{~h}$ after the infusion. Additionally, an oral corticosteroid (e.g. dexamethasone $4 \mathrm{mg}$ ) given post infusion may decrease nausea and vomiting, both increasing tolerability and helping to maintain oral hydration and renal perfusion [47]. After the infusion, the IV line should be flushed with $0.9 \%$ sodium chloride solution to ensure that the entire dose is administered. An additional $1 \mathrm{~L}$ of isotonic IV solution is to be administered as post-infusion hydration, with patients under $50 \mathrm{~kg}$ receiving $0.5 \mathrm{~L}$. In addition to IV hydration, the patient should be advised to take in up to $3 \mathrm{~L}$ (up to $2 \mathrm{~L}$ for patients under $50 \mathrm{~kg}$ ) of oral hydration, such as water, milk, or juice, per day on days 1-8 of each cycle [31].

\section{Toxicities}

The most common adverse reactions (with at least $20 \%$ incidence) include infusion-related reactions, edema, nausea, fatigue, headache, pyrexia, constipation, anemia, and 
diarrhea. Additionally, laboratory abnormalities with at least a $50 \%$ incidence include increased creatinine, increased aspartate aminotransferase and alanine aminotransferase, hypoalbuminemia, hypocalcemia, and hypophosphatemia. Laboratory abnormalities are summarized in Tables 2 and 3. Moxetumomab pasudotox-tdfk carries a boxed warning for CLS and HUS, and warnings and precautions for infusion-related reactions, renal dysfunction, and electrolyte abnormalities.

\section{Hemolytic uremic syndrome}

HUS is characterized by acute kidney injury, thrombocytopenia, and microangiopathic hemolytic anemia. HUS occurred in $7 \%$ of patients treated with moxetumomab pasudotox-tdfk in the combined safety database, including grade 3 in $3 \%$ and grade 4 in $0.8 \%$ of patients [31]. In the phase I trial, grade 2 HUS, defined as anemia with schistocytes and elevated creatinine, developed in two patients (7\%) [45]. In this trial, one patient experienced thrombocytopenia with a platelet nadir of $120,000 / \mu \mathrm{L}$ on day 11 of cycle 3 . The second patient had a platelet nadir of $106,000 / \mu \mathrm{L}$ on day 10 of cycle 5 . The doses administered were $0.03 \mathrm{mg} /$ $\mathrm{kg} /$ dose and $0.05 \mathrm{mg} / \mathrm{kg} /$ dose, respectively. Laboratory abnormalities also included increased creatinine $(1.53 \mathrm{mg} /$ $\mathrm{dL}$ and $1.66 \mathrm{mg} / \mathrm{dL}$ ), lactate dehydrogenase (326 U/L and $238 \mathrm{U} / \mathrm{L})$, and bilirubin $(1.3 \mathrm{mg} / \mathrm{dL}$ and $1.0 \mathrm{mg} / \mathrm{dL})$. In addition, the patient receiving $0.03 \mathrm{mg} / \mathrm{kg} / \mathrm{dose}$ had a decrease in hemoglobin to $10.1 \mathrm{~g} / \mathrm{dL}$. In the pivotal phase 3 trial, HUS occurred in 6 patients $(7.5 \%)$ receiving $0.04 \mathrm{mg} / \mathrm{kg} /$

Table 2 Summary of laboratory abnormalities

\begin{tabular}{lll}
\hline Abnormal value & Percentage of cycles & Grade \\
\hline Hypoalbuminemia & 54 & $1-2$ \\
Elevated AST & 38 & $1-2$ \\
Elevated ALT & 38 & $1-2$ \\
Elevated SCr & $<10$ & $1-2$ \\
GGT & $<10$ & $1-3$ \\
Anemia & $<5$ & 2 \\
Decreased haptoglobin & $<5$ & 3 \\
Leukopenia & $<5$ & 3 \\
Thrombocytopenia & $<5$ & $1-2$ \\
Hyperkalemia & $<5$ & 1 \\
Hyperuricemia & $<5$ & 1 \\
Hypomagnesemia & $<5$ & 1 \\
\hline
\end{tabular}

Phase 1 dose escalation study: $0.005-0.05 \mathrm{mg} / \mathrm{kg} / \mathrm{dose}$ on days 1,3 , and 5; retreatment with $2-16$ additional cycles ( 2 cycles past documentation of CR) [48]

$A L T$, alanine aminotransferase, $A S T$, aspartate aminotransferase, $C R$, complete response, $S C r$, serum creatinine, $G G T$, gamma-glutamyl transferase
Table 3 Summary of laboratory abnormalities

\begin{tabular}{lll}
\hline Abnormal value & $\begin{array}{l}\text { Percentage } \\
\text { of patients of } \\
\text { patients }\end{array}$ & Grades 3/4, \% \\
\hline Hypocalcemia & 23.8 & 0 \\
Hypophosphatemia & 23.8 & 10.0 \\
Anemia & 21.3 & 10.0 \\
Elevated ALT & 21.3 & 1.3 \\
Decreased lymphocyte count & 20.0 & 20.0 \\
Hypoalbuminemia & 20.0 & 0 \\
Hypokalemia & 16.3 & 2.5 \\
Hyponatremia & 11.3 & 2.5 \\
Neutropenia & 5.0 & 5.0 \\
Decreased white blood cell count & 10.0 & 8.8 \\
\hline
\end{tabular}

Phase 3 study: $0.04 \mathrm{mg} / \mathrm{kg} /$ dose on days 1,3 , and 5 for six cycles (maximum six cycles or until documentation of minimal residual disease-negative complete response, progression, or toxicity) [47]

$A L T$, alanine aminotransferase

dose [47]. Permanent discontinuation of moxetumomab pasudotox-tdfk was required in four $(5.0 \%)$ of these patients. No deaths occurred as a result of HUS in prospective trials, and all laboratory abnormalities were reversible and resolved without the use of blood products or plasmapheresis. Most cases of HUS occurred in the first 9 days (range 1-16) of a treatment cycle, but cases have also been reported on other days throughout the cycle. The median time to resolution of HUS was 11.5 days (range 2-44) [31].

Although reversible and manageable, HUS is a serious treatment-related adverse event requiring vigilant monitoring and prophylaxis. Labeled guidance regarding adequate pre- and post-hydration, including oral hydration, is critical in managing fluid and electrolytes and to maintain adequate intravascular volume. Prior to each infusion, hemoglobin, platelet count, and serum creatinine should be assessed, and, if HUS is suspected, LDH, indirect bilirubin, and blood schistocytes should be assessed promptly. Thromboprophylaxis with low-dose aspirin ( $81 \mathrm{mg}$ daily) may be considered on days 1-8 of each 28 -day cycle. In the event a patient develops HUS, treatment should be discontinued [31].

\section{Capillary leak syndrome}

CLS is a syndrome characterized by vascular permeability; signs include hypoalbuminemia, hypotension, and hemoconcentration. Although not unique to moxetumomab pasudotox-tdfk treatment, patients may experience weight gain, ascites, edema, and effusions. CLS causes an intravascular fluid and protein shift that may result in pulmonary edema, transient shock, and death. In addition to hypoalbuminemia, 
laboratory abnormalities include increased hematocrit, leukocytosis, and thrombocytosis.

The incidence of CLS was 34\% in clinical trials, including $23,1.6$, and $2 \%$ with grades 2,3 , and 4 , respectively [31]. Although most cases occurred during the first 8 days of a cycle, the median time to resolution of CLS was 12 days [31]. CLS may occur anytime throughout the cycle. For this reason, patients should be monitored for and counseled to report swelling, weight gain (greater than a 5\% increase), sudden decreased blood pressure, or difficulty breathing to their healthcare provider immediately. If CLS is suspected, check oxygen saturation and evidence of pulmonary edema and/or serosal effusions.

All CLS events resolved with supportive care and/or treatment discontinuation. No deaths from CLS occurred in the clinical trials; however, the condition may be lifethreatening or fatal without proper recognition and treatment. Patients with CLS have intravascular depletion, and judicious use of IV fluids is warranted. Repletion should be gradual, and use of diuretics is not recommended unless the patient is experiencing adverse events related to fluid overload. Other supportive measures include the use of IV or oral corticosteroids. Hospitalization should be considered for all patients suspected of experiencing CLS [31].

\section{Infusion-related reactions}

Infusion-related reactions (IRRs) are a common adverse effect of many immunotherapeutic agents [52]. Despite adequate premedication with an antihistamine, acetaminophen, and a histamine- 2 receptor antagonist, reactions may still occur. Overall, $50 \%$ of patients experienced some form of reaction in the phase 3 trial. Infusion reactions manifested as nausea (15\%), fever (14\%), chills (14\%), vomiting (11\%), and headache (9\%) [31]. In the event of a severe reaction, the infusion should be stopped, and appropriate supportive care should be initiated. If a patient does experience an IRR, a corticosteroid should be administered prior to restarting the infusion and added as a premedication with subsequent infusions [31].

\section{Renal toxicity}

In total, $26 \%$ of patients treated with moxetumomab pasudotox-tdfk experienced renal toxicity, which included acute kidney injury, renal failure, renal impairment, proteinuria, and increased serum creatinine [31]. Serum creatinine was found to increase by two or more grades from baseline ( $22 \%$ of patients), with $1.6 \%$ experiencing a grade 3 increase (greater than 3 times baseline or the upper limit of normal). Five percent of patients experienced a continued elevated serum creatinine 1.5-3 times the upper limit of normal at the end of therapy. Patients older than 65 years, those with pre-existing renal impairment, or those who experienced HUS were at an increased risk of worsening renal function from moxetumomab pasudotox-tdfk. Therapy should be delayed for grade 2 or higher increase (greater than 1.5 times baseline or upper limit of normal) in serum creatinine in patients who have a baseline serum creatinine that is within normal limits. In patients with baseline serum creatinine of grades $1-2$, therapy should be delayed for any serum creatinine increase to grade 3 or higher. Moxetumomab pasudotoxtdfk may be restarted once renal function recovers to baseline or better [31].

\section{Electrolyte abnormalities}

Overall, 57\% of patients experienced electrolyte abnormalities. A summary of laboratory abnormalities from the clinical trials is provided in Tables 2 and 3. The most common abnormality was hypocalcemia, occurring in $25 \%$ of patients overall. Grade 3 and 4 electrolyte abnormalities occurred in $14 \%$ and $0.8 \%$ of patients, respectively. These abnormalities were found to occur during the same treatment cycle as other adverse effects such as CLS, HUS, fluid retention, or renal toxicity just over one-third of the time [31].

\section{Laboratory monitoring and vital signs}

It is important to monitor blood pressure and weight at each visit. Close monitoring of complete blood count and chemistries is recommended prior to and during each cycle of moxetumomab pasudotox-tdfk. Any incidence of anemia, increased creatinine, or thrombocytopenia should be suspected as HUS. If positive, monitoring of indirect bilirubin, LDH, and a peripheral blood smear for schistocytes is warranted. Additionally, patients should be monitored for hypoalbuminemia, weight gain $>5 \%$, hypotension unresponsive to fluids, edema, and shortness of breath, because these can be signs of CLS. If CLS is suspected, check oxygen saturations, and evaluate for pulmonary edema. Although patients may experience generalized edema as a result of moxetumomab pasudotox-tdfk, caution is advised with the use of diuretics in a patient experiencing CLS. Electrolytes should be monitored prior to each infusion and on day 8 of therapy. It is also prudent to monitor electrolytes midway through the cycle. Renal function should be monitored prior to each infusion of moxetumomab pasudotox-tdfk. 


\section{Conclusion}

Moxetumomab pasudotox-tdfk is the first FDA-approved therapy for R/R HCL, and the first new drug approved for HCL since cladribine in 1993. Patients who are no longer responding to alternative therapies or who may not tolerate further treatment with purine analogs owing to myelosuppression or infection may be good candidates for this agent. Moxetumomab pasudotox-tdfk has yielded a durable CR rate of $30 \%$ with a median duration in response that has not yet been reached as of a median follow-up of 16.7 months. The overall response rate was $75 \%$, including $41 \%$ achieving CR and $34 \%$ achieving partial response.

Although each infusion of moxetumomab pasudotox-tdfk is relatively short at $30 \mathrm{~min}$, patients must receive adequate hydration and premedication with an antihistamine, acetaminophen, and a histamine- 2 receptor antagonist to prevent IRRs, HUS, and CLS. To ensure safe and effective use of moxetumomab pasudotox-tdfk, pharmacists and other providers must be vigilant in assessing for these potential events, as well as renal dysfunction and electrolyte abnormalities, and must understand proper methods for the preparation and administration of moxetumomab pasudotox-tdfk.

Funding Editorial support, sponsored by AstraZeneca LP, was provided by Oxford PharmaGenesis, Oxford, UK.

\section{Compliance with ethical standards}

Conflict of interest MJN has participated in an advisory board for Pfizer Inc. AstraZeneca LP reviewed the manuscript for medical accuracy. The authors take responsibility for the manuscript content.

Research involving human participants and/or animals This article does not contain any studies with human participants or animals performed by any of the authors.

Open Access This article is distributed under the terms of the Creative Commons Attribution 4.0 International License (http://creativecommons.org/licenses/by/4.0/), which permits unrestricted use, distribution, and reproduction in any medium, provided you give appropriate credit to the original author(s) and the source, provide a link to the Creative Commons license, and indicate if changes were made.

\section{References}

1. Teras LR, DeSantis CE, Cerhan JR, Morton LM, Jemal A, Flowers CR (2016) 2016 US lymphoid malignancy statistics by world health organization subtypes. CA Cancer J Clin 66(6):443-459

2. Ali S, Saven A (2018) Hairy cell leukemia. In: Wiernik PH, Dutcher JP, Gertz MA (eds) Neoplastic diseases of the blood. Springer, Cham, pp 132-152. https://doi.org/10.1007/978-3-31964263-5_10
3. Flandrin G, Sigaux F, Sebahoun G, Bouffette P (1984) Hairy cell leukemia: clinical presentation and follow-up of 211 patients. Semin Oncol 11(4 Suppl 2):458-471

4. Orsi L, Delabre L, Monnereau A et al (2009) Occupational exposure to pesticides and lymphoid neoplasms among men: results of a french case-control study. Occup Environ Med 66(5):291-298

5. Monnereau A, Slager SL, Hughes AM et al (2014) Medical history, lifestyle, and occupational risk factors for hairy cell leukemia: the InterLymph non-hodgkin lymphoma subtypes project. J Natl Cancer Inst Monogr 2014(48):115-124

6. Stewart DJ, Keating MJ (1980) Radiation exposure as a possible etiologic factor in hairy cell leukemia (leukemic reticuloendotheliosis). Cancer 46(7):1577-1580

7. Else M, Dearden CE, Matutes E et al (2009) Long-term follow-up of 233 patients with hairy cell leukaemia, treated initially with pentostatin or cladribine, at a median of 16 years from diagnosis. Br J Haematol 145(6):733-740

8. Kreitman RJ, Arons E (2018) Update on hairy cell leukemia. Clin Adv Hematol Oncol 16(3):205-215

9. Matutes E, Martinez-Trillos A, Campo E (2015) Hairy cell leukaemia-variant: disease features and treatment. Best Pract Res Clin Haematol 28(4):253-263

10. Bartl R, Frisch B, Hill W, Burkhardt R, Sommerfeld W, Sund M (1983) Bone marrow histology in hairy cell leukemia. Identification of subtypes and their prognostic significance. Am J Clin Pathol 79(5):531-545

11. National Comprehensive Cancer Network. Hairy cell leukemia (version 1.2019). https://Www.nccn.org/professionals/physician_ gls/pdf/hairy_cell.pdf.

12. Shao H, Calvo KR, Gronborg M et al (2013) Distinguishing hairy cell leukemia variant from hairy cell leukemia: development and validation of diagnostic criteria. Leuk Res 37(4):401-409

13. Durham BH, Getta B, Dietrich S et al (2017) Genomic analysis of hairy cell leukemia identifies novel recurrent genetic alterations. Blood 130(14):1644-1648. https://doi.org/10.1182/blood-201701-765107

14. Grever MR, Abdel-Wahab O, Andritsos LA et al (2017) Consensus guidelines for the diagnosis and management of patients with classic hairy cell leukemia. Blood 129(5):553

15. Spiers ASD, Moore D, Cassileth PA et al (1987) Remissions in hairy-cell leukemia with pentostatin ( $2^{\prime}$-deoxycoformycin). N Engl J Med 316(14):825-830

16. Grever M, Kopecky K, Foucar MK et al (1995) Randomized comparison of pentostatin versus interferon alfa-2a in previously untreated patients with hairy cell leukemia: an intergroup study. J Clin Oncol 13(4):974-982

17. Flinn IW, Kopecky KJ, Foucar MK et al (2000) Long-term follow-up of remission duration, mortality, and second malignancies in hairy cell leukemia patients treated with pentostatin. Blood 96(9):2981

18. Goodman GR, Burian C, Koziol JA, Saven A (2003) Extended follow-up of patients with hairy cell leukemia after treatment with cladribine. J Clin Oncol 21(5):891-896

19. Chihara D, Kantarjian H, O'Brien S et al (2016) Long-term durable remission by cladribine followed by rituximab in patients with hairy cell leukaemia: update of a phase II trial. Br J Haematol 174(5):760-766

20. Else M, Dearden CE, Matutes E et al (2011) Rituximab with pentostatin or cladribine: an effective combination treatment for hairy cell leukemia after disease recurrence. Leuk Lymphoma 52(Suppl 2):75-78

21. Lauria F, Lenoci M, Annino L et al (2001) Efficacy of anti-CD20 monoclonal antibodies (mabthera) in patients with progressed hairy cell leukemia. Haematologica 86(10):1046-1050

22. Zenhausern R, Simcock M, Gratwohl A et al (2008) Rituximab in patients with hairy cell leukemia relapsing after treatment 
with 2-chlorodeoxyadenosine (SAKK 31/98). Haematologica 93(9):1426-1428

23. Thomas DA, O'Brien S, Bueso-Ramos C et al (2003) Rituximab in relapsed or refractory hairy cell leukemia. Blood 102(12):3906-3911

24. Nieva J, Bethel K, Saven A (2003) Phase 2 study of rituximab in the treatment of cladribine-failed patients with hairy cell leukemia. Blood 102(3):810-813

25. Tiacci E, Park JH, De Carolis L et al (2015) Targeting mutant BRAF in relapsed or refractory hairy-cell leukemia. N Engl J Med 373(18): 1733-1747

26. Tiacci E, De Carolis L, Zaja F et al (2017) The chemotherapy-free combination of vemurafenib and rituximab produces deep and durable responses in relapsed or refractory hairy cell leukemia (HCL) patients. Blood 130(Suppl 1):409

27. Jones J, Andritsos L, Kreitman RJ et al (2016) Efficacy and safety of the bruton tyrosine kinase inhibitor ibrutinib in patients with hairy cell leukemia: stage 1 results of a phase 2 study. Blood 128(22): 1215

28. Mhawech-Fauceglia P, Oberholzer M, Aschenafi S et al (2006) Potential predictive patterns of minimal residual disease detected by immunohistochemistry on bone marrow biopsy specimens during a long-term follow-up in patients treated with cladribine for hairy cell leukemia. Arch Pathol Lab Med 130(3):374-377

29. Ravandi F, Jorgensen JL, O'Brien SM et al (2006) Eradication of minimal residual disease in hairy cell leukemia. Blood 107(12):4658-4662

30. Tiacci E, De Carolis L, Zaja F et al (2016) Vemurafenib plus rituximab in hairy cell leukemia: a promising chemotherapy-free regimen for relapsed or refractory patients. Blood 128(22):1214

31. LUMOXITI [package insert]. Wilmington, DE: AstraZeneca Pharmaceuticals LP, 2018.

32. Aruna G (2006) Immunotoxins: a review of their use in cancer treatment. J Stem Cells Regen Med 1(1):31-36

33. Kreitman RJ, Pastan I (2015) Immunoconjugates in the management of hairy cell leukemia. Best Pract Res Clin Haematol. 28(4):236-245

34. Pastan I, Hassan R, Fitzgerald DJ, Kreitman RJ (2006) Immunotoxin therapy of cancer. Nat Rev Cancer 6(7):559-565

35. Tedder TF, Tuscano J, Sato S, Kehrl JH (1997) CD22, a B lymphocyte-specific adhesion molecule that regulates antigen receptor signaling. Annu Rev Immunol 15:481-504

36. Lajaunias F, Nitschke L, Moll T et al (2002) Differentially regulated expression and function of CD22 in activated B-1 and B-2 lymphocytes. J Immunol 168(12):6078-6083

37. Nitschke L, Carsetti R, Ocker B, Kohler G, Lamers MC (1997) $\mathrm{CD} 22$ is a negative regulator of B-cell receptor signalling. Curr Biol 7(2):133-143

38. Erickson LD, Tygrett LT, Bhatia SK, Grabstein KH, Waldschmidt TJ (1996) Differential expression of CD22 (Lyb8) on murine B cells. Int Immunol 8(7):1121-1129

39. Shan D, Press OW (1995) Constitutive endocytosis and degradation of CD22 by human B cells. J Immunol 154(9):4466-4475

40. Moyron-Quiroz JE, Partida-Sanchez S, Donis-Hernandez R, Sandoval-Montes C, Santos-Argumedo L (2002) Expression and function of CD22, a B-cell restricted molecule. Scand J Immunol 55(4):343-351

41. Sieger N, Fleischer SJ, Mei HE et al (2013) CD22 ligation inhibits downstream B cell receptor signaling and $\mathrm{ca}(2+)$ flux upon activation. Arthritis Rheum 65(3):770-779
42. Naeim F, Song SX, Grody WW (2012) Atlas of hematopathology: Morphology, immunophenotype, cytogenetics, and molecular approaches. Elsevier, San Diego

43. Alderson RF, Kreitman RJ, Chen T et al (2009) CAT-8015: a second-generation pseudomonas exotoxin A-based immunotherapy targeting CD22-expressing hematologic malignancies. Clin Cancer Res 15(3):832-839

44. Salvatore G, Beers R, Margulies I et al (2002) Improved cytotoxic activity toward cell lines and fresh leukemia cells of a mutant anti-CD22 immunotoxin obtained by antibody phage display. Clin Cancer Res 8(4):995-1002

45. Kreitman RJ, Tallman MS, Robak T et al (2012) Phase I trial of anti-CD22 recombinant immunotoxin moxetumomab pasudotox (CAT-8015 or HA22) in patients with hairy cell leukemia. J Clin Oncol 30(15): 1822-1828

46. Kreitman RJ, Pastan I (2011) Antibody fusion proteins: anti-CD22 recombinant immunotoxin moxetumomab pasudotox. Clin Cancer Res 17(20):6398-6405

47. Kreitman RJ, Dearden C, Zinzani PL et al (2018) Moxetumomab pasudotox in relapsed/refractory hairy cell leukemia. Leukemia 32(8):1768-1777

48. Kreitman RJ, Tallman MS, Robak T et al (2018) Minimal residual hairy cell leukemia eradication with moxetumomab pasudotox: phase 1 results and long-term follow-up. Blood 131(21):2331-2334

49. Tallman MS, Hakimian D, Kopecky KJ et al (1999) Minimal residual disease in patients with hairy cell leukemia in complete remission treated with 2-chlorodeoxyadenosine or 2-deoxycoformycin and prediction of early relapse. Clin Cancer Res 5(7):1665-1670

50. Wheaton S, Tallman MS, Hakimian D, Peterson L (1996) Minimal residual disease may predict bone marrow relapse in patients with hairy cell leukemia treated with 2-chlorodeoxyadenosine. Blood 87(4):1556-1560

51. Arons E, Gorelik D, Stetler-Stevenson M et al (2018) Molecular remissions with anti-CD22 recombinant immunotoxin moxetumomab pasudotox are associated with improved complete remission durations during phase I and III testing. Blood 132(Suppl 1):1861. https://doi.org/10.1182/blood-2018-99-113419

52. Patel SV, Khan DA (2017) Adverse reactions to biologic therapy. Immnol Allergy Clin North Am 37(2):397-412

53. Estey EH, Kurzrock R, Kantarjian HM et al (1992) Treatment of hairy cell leukemia with 2-chlorodeoxyadenosine (2-CdA). Blood 79(4):882-887

54. Saven A, Burian C, Koziol JA, Piro LD (1998) Long-term followup of patients with hairy cell leukemia after cladribine treatment. Blood 92(6): 1918-1926

55. Chadha P, Rademaker AW, Mendiratta P et al (2005) Treatment of hairy cell leukemia with 2-chlorodeoxyadenosine (2-CdA): longterm follow-up of the northwestern university experience. Blood 106(1):241

Publisher's Note Springer Nature remains neutral with regard to jurisdictional claims in published maps and institutional affiliations. 